\title{
Diagnosis and treatment of early gastric cancer: experience of one center
}

\author{
Zbigniew K. Kula ${ }^{1}$, Wojciech Zegarski², Wojciech Jóźwicki \\ ${ }^{1}$ Department of Endoscopy, F. Łukaszczyk Oncology Center, Bydgoszcz, Poland \\ 2Department of Oncological Surgery, Ludwik Rydygier Collegium Medicum in Bydgoszcz, Nicolaus Copernicus University \\ in Torun, F. Łukaszczyk Oncology Center, Bydgoszcz, Poland \\ ${ }^{3}$ Department of Tumor Pathology and Pathomorphology, Ludwik Rydygier Collegium Medicum in Bydgoszcz, \\ Nicolaus Copernicus University in Torun, F. Łukaszczyk Oncology Center, Bydgoszcz, Poland
}

Gastroenterology Rev 2018; 13 (3): 200-205

DOI: https://doi.org/10.5114/pg.2018.73332

Key words: early gastric cancer, endoscopy, treatment.

Address for correspondence: Zbigniew K. Kula MD, PhD, Department of Endoscopy, F. Łukaszczyk Oncology Center, 2 Romanowskiej St, 85-795 Bydgoszcz, Poland, phone: +48 5238139 12, +48 606464 455, e-mail: zbigniew.kula@co.bydgoszcz.pl

\begin{abstract}
Introduction: Gastric cancer is one of the most common malignant neoplasms in the world. Currently it constitutes the third cause of death among all malignancies. New endoscopic techniques have a potential to improve treatment results due to more frequent detection of early gastric cancer.

Aim: To summarize our experience in diagnosis and treatment of early gastric cancer patients.

Material and methods: We analyzed the results of endoscopic examination, histopathological findings and treatment methods in 16 patients who were diagnosed with early gastric cancer in the Endoscopy Unit of the Oncology Center in Bydgoszcz between 2014 and 2016.

Results: Between 2014 and 2016 sixteen patients, 12 (75.0\%) male and 4 (25.0\%) female, were diagnosed with early gastric cancer. The average age of patients was 65.5 years. Surgery qualification concerned mainly G2 and G3 adenocarcinomas. In $12(75.0 \%)$ patients total gastrectomy was performed, and 1 patient underwent wedge resection of the prepyloric part of the stomach. In 3 cases endoscopic submucosal dissection (ESD) was performed. Histopathological examination of both biopsy and postoperative material indicated 8 (50.0\%) cases of intestinal-type adenocarcinoma, 7 (43.75\%) cases of diffuse-type adenocarcinoma and 1 (6.25\%) case of mixed-type adenocarcinoma. During the follow-up after treatment, none of the patients was diagnosed with local or distant recurrence.

Conclusions: Accurate examination technique allows for detection of early gastric cancer. Selected cases of early gastric cancer may be treated with advanced endoscopic techniques.
\end{abstract}

\section{Introduction}

Gastric cancer is still one of the most common malignant neoplasms in the world. Currently it constitutes the third cause of death among all malignancies. In spite of the progress of surgical methods and chemotherapy, the 5-year survival rate among patients in Europe and the USA does not exceed 30\% [1, 2]. Development of new endoscopic techniques has a potential to improve treatment results due to detection of early gastric cancer. Early cancers (T1) of the gastrointestinal tract are neoplasms restricted to mucosa (Tm1) and submucosa (T1sm). Information about the depth of early cancer invasion and the status of regional lymph node involvement is essential for the prognosis. According to the Japanese classification, there are 3 types of early gastric cancer: type I - polypoid, type II - superficial, and type III - ulcerative [3]. Type II is divided into three subtypes, i.e. Ila - superficial elevated, Ilb - superficial flat (at the level of mucosa), and IIc - superficial depressed (slightly depressed). In Europe the most frequently used classification of superficial neoplastic lesions is the Paris classification [4]. The highest rate of early gastric cancer detection has been reported in such countries as Japan (50\%) and Korea (46-67\%). In Europe it is significantly lower (15\%) [5-7]. In Poland it has 
not been estimated. An improvement in the detection rate of early proliferative lesions within the stomach depends on several factors including the individual skills of physicians and imaging techniques they use as well as the health awareness of society and implementation of screening programs.

\section{Aim}

The aim of this study was to summarize our experience in diagnosis and treatment of early gastric cancer patients.

\section{Material and methods}

We analyzed the results of endoscopic examination, histopathological findings and treatment methods in 16 patients who were diagnosed with early gastric cancer in the Endoscopy Unit of the Oncology Center in Bydgoszcz between 2014 and 2016. Endoscopic examinations were performed with Olympus systems (GIF-Q180, GIF-QTH180 and GIF-Q190). White-light and narrow-band imaging (NBI) was used. Photodocumentation was stored in the Olympus Endobase system. The preliminary diagnosis was established on the basis of the endoscopic image that served as a reference. For histopathological analysis 2 to 4 specimens were collected from the suspicious gastric lesion. All patients with pathologic diagnoses of adenocarcinoma underwent contrast-enhanced abdominal and chest computed tomography (CT). In 3 cases endoscopic ultrasound examination was performed. All patients were qualified for treatment by the Gastric Cancer Treatment Interdisciplinary Team consisting of a surgeon, clinical oncologist, radiologist, pathologist, radiotherapist and gastroenterologist [8]. The final diagnosis was established on the basis of histopathological findings obtained from surgical and endoscopic specimens. Cancer staging was performed based on pathological examination in accordance with the current TNM classification. Patients who underwent endoscopic submucosal dissection (ESD) had a follow-up endoscopic examinations every 3 months for 2 years. In the case of patients after surgical intervention endoscopic examination was performed every 12 months. Once a year abdominal CT was performed.

\section{Results}

Between 2014 and 2016, 16 patients, 12 (75.0\%) male and 4 (25.0\%) female, were diagnosed with early gastric cancer (Table I, Figure 1). The average age of all patients was 65.5 years, with the average of 67.5 years in women and 64.8 years in men. In 11 (68.7\%) patients dyspeptic symptoms were present and they were the most frequently reported complaints. Five (31.25\%) patients were asymptomatic. Location of early gastric cancer included gastric body (6 patients $-37.0 \%$ ), prepyloric part of stomach (4 patients $-25.0 \%$ ), gastric angle (3 patients - 18.75\%) and gastric cardia (3 patients $-18.75 \%$ ). A surgical approach was used in the majority of cases. The surgery qualification included mainly adenocarcinomas with histological differentiation grades $\mathrm{G} 2$ and $\mathrm{G} 3$. In 12 (75.0\%) patients total gastrectomy was performed. One patient underwent wedge resection of the prepyloric part of the stomach. During surgical procedures performed in 13 patients a total of 231 lymph nodes were removed (on average of 17.8 nodes per procedure) and assessed as free of metastasis. In the postoperative period 1 patient experienced anastomosis leak that was successfully treated using a temporal self-expandable prosthesis. In 3 other patients ESD was performed. No complications were observed after endoscopic treatment. The endoscopic assessment results and histopathological findings were consistent in terms of lesion size and submucosal infiltration degree. It was also confirmed that the endoscopic assessment of all cases treated surgically matched the result obtained during the postoperative material examination. The histopathological examination of both biopsy and postoperative material indicated $8(50.0 \%)$ cases of intestinal-type adenocarcinoma, 7 (43.75\%) cases of diffuse-type adenocarcinoma and $1(6.25 \%)$ case of mixed-type adenocarcinoma. In $7(43.75 \%)$ cases the degree of the histological maturity of the cancer was classified as G3, in 5 (31.25\%) cases as $G 2$ and in 4 (25.00\%) patients as G1. During the follow-up none of the patients was diagnosed with local or distant recurrence.

\section{Discussion}

The advances in endoscopic technology seen in recent decades and better understanding of gastric cancer pathophysiology of the gastrointestinal tract have contributed to more frequent detection and earlier treatment. The difference in early gastric cancer detection rate that is observed between Japan and Europe may be associated with diverse endoscopic and histopathological examination protocols. In their collective analysis Menon and Trudgill [9] proved that $11.3 \%$ of cancers located in the upper gastrointestinal tract may be overlooked in gastroscopy that was performed up to 3 years before the diagnosis was made. In order to improve the effectiveness of gastroscopy it is necessary to perform full air insufflation, remove the mucus and foam through gastric irrigation, and keep records of photodocumentation and biopsy protocols. The examination time should be extended, especially in patients 


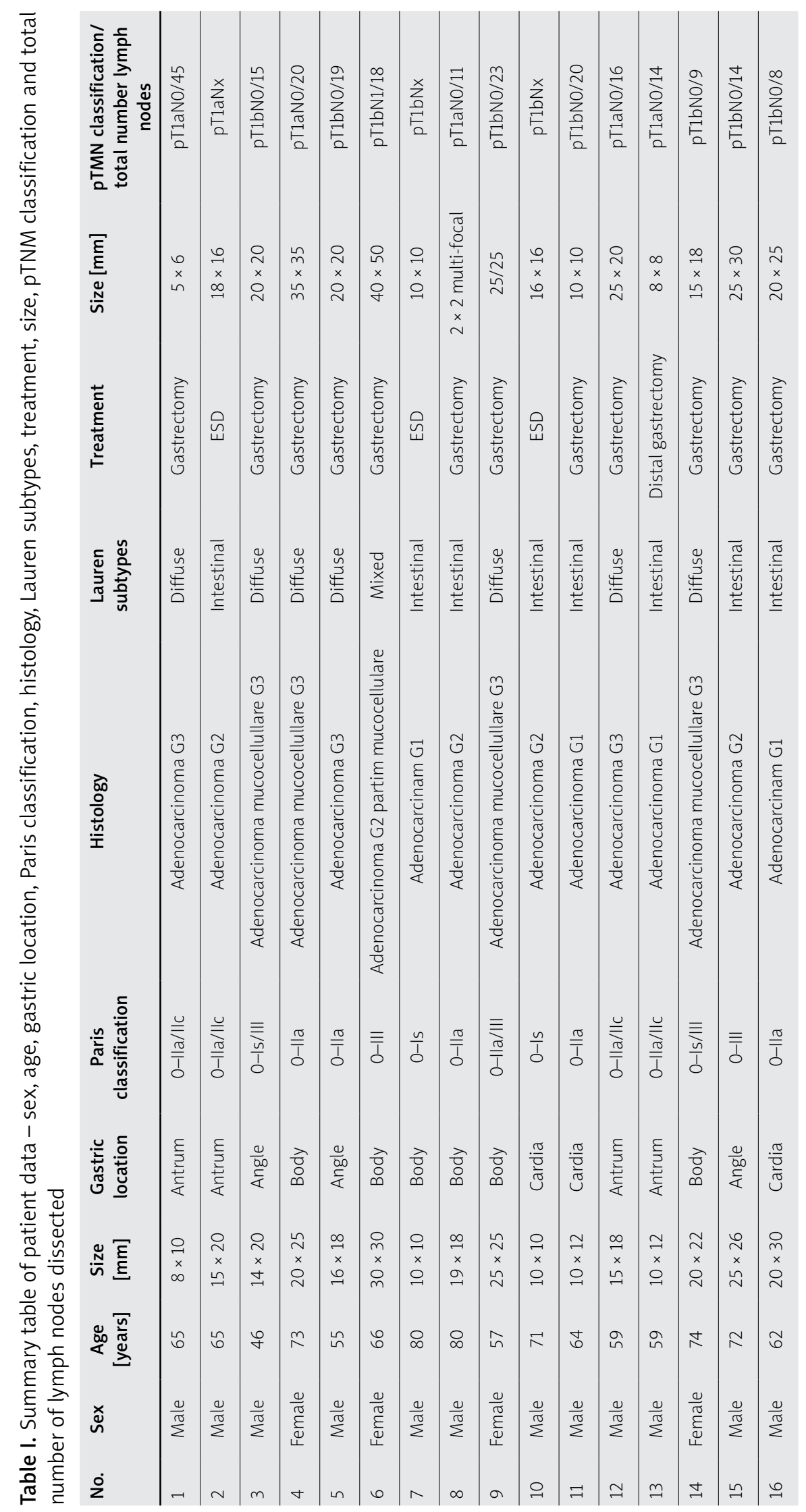




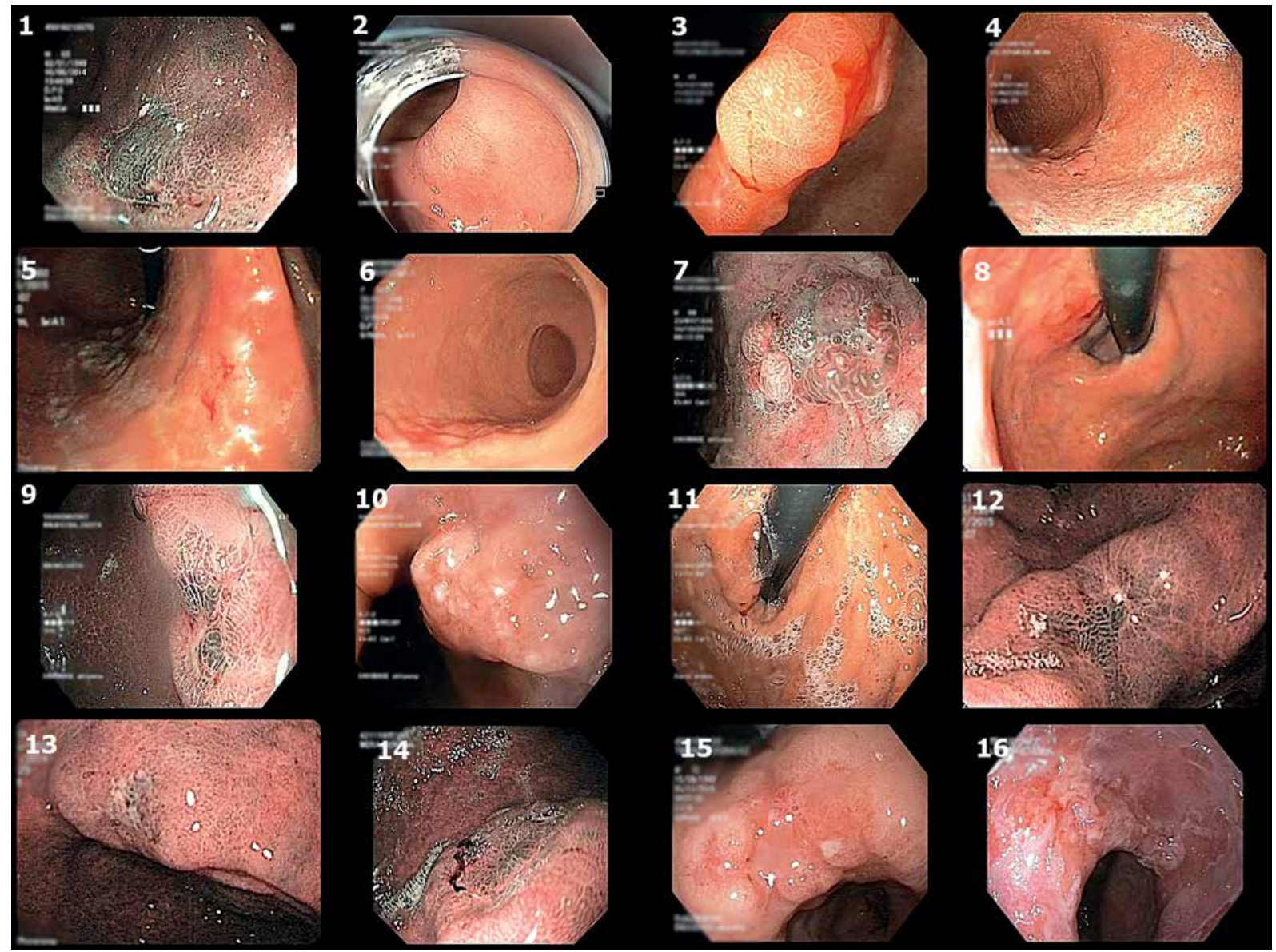

Figure 1. Endoscopic images of early gastric cancer. Conventional white light $(2-6,8,10,11,15,16)$ and narrow-band imaging endoscopy $(1,7,9,12-14)$

showing signs of inflammation and metaplasia that increase the risk of neoplasia. If more than $7 \mathrm{~min}$ are allowed for gastroscopy, the detection rate of high-risk lesions, dysplasia and gastric cancer increases [10]. The Asian SSS protocol (Systematic Screening protocol for the Stomach) suggests preparing documentation consisting of at least 22 representative images, which in case of the ESGE guidelines are limited to 10 [11-13].

During endoscopic examination of our patients we paid particular attention to the assessment of the mucosal surface. We searched for elevations and depressions, changes in color and vascular abnormalities, as well as spontaneous and contact bleedings. We used NBI to ensure more accurate assessment of the surface and vascularization of lesions found during endoscopy. Yao [12] proposed a simple VS (Vascular and Surface pattern) classification in the case of such superficial lesions. Finding both microsurface pattern irregularities (surface - S) and microvascular pattern irregularities (vessel - V) increases the sensitivity (95\%) and specificity (97\%) of early cancer detection. The use of a simple GUP (gastritis-like lesions $(\mathrm{G})$, ulcerative lesions $(\mathrm{U})$ and polypoid lesions (P)) system is also suggested for early gastric cancer diagnosis and characterization of the endoscopic lesions found. This system distinguishes gastritis-like lesions, ulcerative lesions and polypoid lesions [12].

Another classification used in the course of assessing mucosal lesions suspected of cancerous hyperplasia was the Paris classification. It was used mainly to assess the risk of submucosal invasion. It is assumed that the highest percentage of submucosal layer infiltration occurs in superficial neoplastic lesions of type $0-1$ and 0-Ila + IIc. If ulceration, significant elevation of the lesion and fold convergence with elevation of the whole region were observed, preliminary diagnosis indicated infiltration or excess of deeper layers of submucosa.

The extent of gastrectomy and lymphadenectomy in gastric cancer, as well as the recommended reconstruction methods, has regularly evolved in recent years [14]. Total gastrectomy with lymphadenectomy for years has remained the standard of care in the radical treatment of gastric cancer. Partial or subtotal gastrectomy is accepted in selected cases of peripheral tumor location and early gastric cancer [15]. 
Until recently surgical treatment of gastric cancer was considered the only curative method. This view changed when analyses were performed for early gastric cancer patients after gastrectomy and lymphadenectomy. In these cases the 5-year survival rate was higher than $90 \%$, and the lymph node metastasis rate for $\mathrm{T} 1 \mathrm{a}$ and $\mathrm{T} 1 \mathrm{~b}$ stages was $3 \%$ and $20 \%$, respectively [16]. These results of these analyses and the determined perioperative morbidity rate in the order of a few percent suggest that surgery may not be the correct approach in the case of early gastric cancer. Having that in mind, in selected cases of early gastric cancer low-invasive procedures, such as wedge resection and/ or advanced endoscopic techniques, may constitute an equally radical treatment method.

Endoscopic resection of superficial neoplastic lesions of the gastrointestinal tract is becoming a more and more common treatment method with efficacy comparable to surgical treatment. The advantages of endoscopic methods include low invasiveness, a smaller number of complications, lower treatment costs and better post-procedure quality of life. The first endoscopic mucosal resections (EMR) were performed by the Japanese at the beginning of the 1980s. Further dynamic development of mucosal resection methods is associated with the introduction of endoscopic knife (IT Knife, insulation-tipped electrosurgical knife) in 1996, which allowed for one-piece resection (en bloc) of the affected mucosa in the field of $>2 \mathrm{~cm}$ in diameter [16-18]. This type of procedure (ESD) has an advantage over EMR because it gives the possibility to pathologically assess the oncological radicality of the resected lesion together with the horizontal and vertical margin of normal tissue and offers a low percentage of local recurrence in comparison to EMR [16].

Neoplastic lesions of the gastrointestinal tract that have a very small risk of lymph node metastasis and may be resected en bloc are the general indication for endoscopic resection. Specific indications change every few years. These changes are driven by the advances observed in this field - development of endoscopic imaging techniques, introduction of new endoscopic tools and analyzing treatment results obtained in an increasing number of patients. The European Society of Gastrointestinal Endoscopy (ESGE) recommends considering endoscopic treatment of gastric lesions in the following cases: dysplastic lesions regardless of their size, intramucosal differentiated-type adenocarcinoma without ulceration (size $\leq 2 \mathrm{~cm}$, > $2 \mathrm{~cm}$ expanded indication), intramucosal differentiated-type adenocarcinoma with ulceration (size $\leq 3 \mathrm{~cm}$, expanded indication), intramucosal undifferentiated adenocarcinoma (size $\leq 2 \mathrm{~cm}$, expanded indication), differentiated-type adenocarcinoma with submucosal invasion ( $\mathrm{sm} 1 \leq 500 \mu \mathrm{m}$, size $\leq 3 \mathrm{~cm}$, expanded indication) [18].

The Japanese Gastric Cancer Association (JGCA) recommends endoscopic treatment (EMR or ESD) as a standard treatment for differentiated-type adenocarcinomas without ulceration, $\mathrm{T} 1 \mathrm{a}$, size $\leq 2 \mathrm{~cm}$. Radical procedure criteria are met under the following conditions: en bloc resection, size $\leq 2 \mathrm{~cm}$, poorly differentiated adenocarcinoma, pT1a stage, negative horizontal and vertical margins, no lymphovascular infiltration. Expanded indications include cancer cases classified as T1a and assessed as highly differentiated without ulceration $>2 \mathrm{~cm}$ in diameter, highly differentiated with ulceration $\leq 3 \mathrm{~cm}$ in diameter and poorly differentiated without ulceration $\leq 2 \mathrm{~cm}$ in diameter [19].

In the material presented, the initial ESD qualification was positive for 5 patients, in the case of whom the endoscopic image did not reveal neoplastic infiltration extending beyond submucosa or the risk of submucosal invasion assessed in accordance with the Paris classification was low. In 2 cases we did not perform dissection due to the fact that the lesion did not lift after submucosal injection of indigo carmine solution. In 1 case the lesion, although superficial, did not lift probably because of cancerous hyperplasia within the atrophic mucosa and a very thin layer of the submucosa. In addition, this lesion displayed contact and spontaneous bleeding. In the remaining cases ESD was radical and was performed without complications. The pathological assessment of the endoscopic material resected en bloc and postoperative material confirmed that it is possible to assess the submucosal invasion on the basis of the endoscopic image. During the 2-year follow-up after endoscopic treatment local recurrence or lymph node metastases were not identified.

The good outcomes of surgical treatment and low percentage of complications observed in our material show how important the role of the operating surgeon is. Surgery performed in a highly specialized department by experienced physicians is probably the key factor reducing the risk of postoperative complications and consequently leading to better outcomes of gastric cancer treatment. In facilities having limited experience in advanced endoscopic techniques gastrectomy should remain the gold standard of early gastric cancer treatment [20].

It should be pointed out that the correct multi-specialty treatment of early gastric cancer is not possible unless the histological type is determined together with its differentiation level and clinical stage. The most important step of the process is the identification of the treatment method used by a multi-specialty team. 


\section{Conclusions}

Accurate examination technique and use of new generation endoscopes allow for detection of early gastric neoplastic lesions. The model of multi-specialty qualification for treatment, including advanced endoscopic techniques, should be promoted.

\section{Conflict of interest}

The authors declare no conflict of interest.

\section{References}

1. Berrino F, De Angelis R, Sant M, et al. EUROCARE Working Group. Survival for eight major cancer and all cancers combined for European adults diagnosed in 1995-1999: results of EUROCARE-4 study. Lancet Oncol 2007; 8: 773-83.

2. Marques-Lespier JM, Gonzalez-Pons M, Cruz-Correa M. Current perspectives on gastric cancer. Gastroenterol Clin North Am 2016; 45: 413-28.

3. Japanese Research Society for Gastric Cancer. Japanese classification of gastric carcinoma. First English Edition. Kanehara \& Co, Ltd. Tokyo 1995.

4. The Paris endoscopic classification of superficial neoplastic lesions: esophagus, stomach, and colon. November 30 to December 1, 2002. Participants in the Paris Workshop. Gastrointest Endosc 2003; 58 (suppl): S3-43.

5. Shimizu S, Tada M, Kawai K. Early gastric cancer: its surveillance and natural course. Endoscopy 1995; 27: 27-31.

6. Noguchi Y, Yoshhikawa T, Tsuburaya A, et al. Is gastric cancer different between Japan and the United States? Cancer 2000; 89: 2237-46.

7. Lee H, Min BH, Lee JH, et al. Survival outcome associated with the screening interval for gastric cancer in Korea. Digestion 2011; 84: 142-48.

8. Polkowski WP. Rak żołądka - rola gastroenterologa w zespotach wielodyscyplinarnych. Gastroentrol Klin 2014; 6: 172-9.

9. Menon S, Trudgill N. How commonly is upper gastrointestinal cancer missed at endoscopy? A meta-analysis. Endosc Int Open 2014; 2: E46-50.

10. Teh JL, Tan JR, Lau LJF. Longer examination time improves detection of gastric cancer during diagnostics upper gastrointestinal endoscopy. Clin Gastroenterol Hepatol 2015; 13: 480-87.

11. Yao K. The endoscopic diagnosis of early gastric cancer. Ann Gastroenterol 2013; 26: 11-22.

12. Yao K. Development of an e-learning system for teaching endoscopists how to diagnose early gastric cancer: basic principles for improving early detection. Gastric Cancer 2017; 20: S28-38.

13. Bisschops R, Areia M, Coron E. Performance measures for upper gastroitestinal endoscopy: a European Society of Gastrointestinal Endoscopy (ESGE) Quality Improvement Initiative. Endoscopy 2016; 48: 843-64.

14. Olesiński T. Chirurgiczne leczenie raka żołądka dawniej i dziś. Nowotwory 2016; 1: 164-71.

15. Sano T, Kobori O, Muto T. Lymph node metastasis from early gastric cancer: endoscopic resection of tumor. Br J Surg 1992; 79: 241-4.
16. Ono H. Early gastric cancer: diagnosis, pathology, treatment techniques and treatment outcomes. Eur J Gastroenterol Hepatol 2006; 18: 863-6.

17. Gotoda T, Jung HY. Endoscopic resection (endoscopic mucosal resection/endoscopic submucosal dissection) for early gastric cancer. Digestive Endoscopy 2013; 25 (Supp. 1): 55-63.

18. Pimentel-Nunes P, Dinis-Ribeiro M, Ponchon T, et al. Endoscopic submucosa dissection: European Society of Gastrointestinal Endoscopy (ESGE) Guideline. Endoscopy 2015; 47: 829-54.

19. Japanese Gastric Cancer Association. Japanese gastric cancer treatment guidelines 2014 (ver.4). Gastric Cancer 2017; 20: 1-19.

20. Montgomery M, Fukuhara S, Karpeh M, et al. Evidence-based review of the management of early gastric cancer. Gastroenterol Rep 2013; 1: 105-12.

Received: 17.12 .2017

Accepted: 1.01 .2018 